\title{
A novel ALMS1 splice mutation in a non-obese juvenile-onset insulin-dependent syndromic diabetic patient
}

\author{
May Sanyoura ${ }^{1,2}$, Cédric Woudstra ${ }^{1,2}$, George Halaby ${ }^{3}$, Patrick Baz ${ }^{4}$, Valérie Senée ${ }^{1,2}$, Pierre-Jean \\ Guillausseau $^{1,2,5}$, Pierre Zalloua ${ }^{6}$ and Cécile Julier ${ }^{\star, 1,2}$
}

Insulin-dependent juvenile-onset diabetes may occur in the context of rare syndromic presentations suggesting monogenic inheritance rather than common multifactorial autoimmune type 1 diabetes. Here, we report the case of a Lebanese patient diagnosed with juvenile-onset insulin-dependent diabetes presenting ketoacidosis, early-onset retinopathy with optic atrophy, hearing loss, diabetes insipidus, epilepsy, and normal weight and stature, who later developed insulin resistance. Despite similarities with Wolfram syndrome, we excluded the WFS1 gene as responsible for this disease. Using combined linkage and candidate gene study, we selected ALMS1, responsible for Alström syndrome, as a candidate gene. We identified a novel splice mutation in intron 18 located 3 bp before the intron-exon junction (IVS18-3T $>$ G), resulting in exon 19 skipping and consequent frameshift generating a truncated protein (V3958fs3964X). The clinical presentation of the patient significantly differed from typical Alström syndrome by the absence of truncal obesity and short stature, and by the presence of ketoacidotic insulin-dependent diabetes, optic atrophy and diabetes insipidus. Our observation broadens the clinical spectrum of Alström syndrome and suggests that ALMS1 mutations may be considered in patients who initially present with an acute onset of insulin-dependent diabetes.

European Journal of Human Genetics (2014) 22, 140-143; doi:10.1038/ejhg.2013.87; published online 8 May 2013

Keywords: diabetes; genetic diagnosis; monogenic disease; Wolfram syndrome; Alström syndrome

\section{INTRODUCTION}

The majority of patients with juvenile-onset insulin-dependent diabetes are diagnosed as type 1 diabetes (T1D), an autoimmune disease characterized by absolute deficiency in insulin secretion. ${ }^{1}$ However, $\sim 10 \%$ of patients diagnosed as T1D do not have detectable diabetes-specific autoantibodies at disease onset, suggesting that the disease mechanism in some patients may be non-autoimmune, and the possibility of genetic heterogeneity within T1D. A subset of autoantibody-negative diabetes may have a monogenic cause, including Maturity-Onset Diabetes of the Young (MODY), neonatal diabetes, ${ }^{2}$ and other diabetes subtypes. In particular, we previously showed that homozygous mutations in the WFS1 gene are frequent monogenic causes of juvenile-onset insulindependent diabetes in Lebanon, resulting in Wolfram syndrome (WFS) or non-syndromic diabetes diagnosed as T1D. ${ }^{3}$ In practice, some of the characteristic features of monogenic forms of diabetes may be missed or ignored at the time of diagnosis, resulting in an incorrect diagnosis, which is further complicated by clinical overlap between different forms of monogenic diabetes.

Here, we used a comprehensive genetic strategy to investigate a patient who was initially diagnosed as WFS based on clinical presentation, and we identified a novel homozygous mutation in the ALMS1 gene responsible for diabetes in this patient.

\section{SUBJECTS AND METHODS}

\section{Patient}

The patient, a Lebanese boy, was diagnosed with T1D at the age of 13, with severe ketoacidosis at onset. He also had progressive vision loss with optic atrophy, bilateral hearing loss and neurological manifestations. The patient and his parents signed an informed consent to participate in the genetic study, which was approved by the Research and Ethics Committee of the Chronic Care Center, Lebanon. Detailed clinical and biological examinations were done at age 19, as described. ${ }^{4}$ DNA from 180 Lebanese subjects was used as population controls. ${ }^{5}$

\begin{abstract}
Methods
Blood samples were collected from the patient and his parents, and genomic DNA was extracted from peripheral blood leukocytes using standard protocols. Total RNA was extracted from blood from both parents using the PAXgene Blood RNA System Kit (Qiagen, Courtaboeuf, France), and cDNA was synthesized using oligo(dT) primers and SuperScript III Reverse Transcriptase (Invitrogen, Cergy-Pontoise, France). Sequencing of the exons and exonintron boundaries of the WFS1 and ALMS1 genes was performed after PCR amplification of genomic DNA, with specific primers for $W F S 1^{3}$ and ALMS1. Sequences were interpreted using the Genalys software. ${ }^{6}$

A microsatellite genome scan was performed using $>400$ microsatellites, complemented by three microsatellite markers near WFS1. ${ }^{3}$ Linkage study was performed under a highly penetrant recessive model (penetrance: 1 , disease allele frequency: 0.001 , no phenocopy), using MERLIN package. ${ }^{7}$ Splice site
\end{abstract}

${ }^{1}$ Inserm UMR-S958, Medical Faculty Paris 7, site Villemin, Paris, France; ${ }^{2}$ University Paris 7 Denis-Diderot, Paris, France; ${ }^{3}$ Division of Endocrinology, Hôtel Dieu Hospital, Beirut, Lebanon; ${ }^{4}$ Department of Ophthalmology, Hôtel Dieu Hospital, Beirut, Lebanon; ${ }^{5}$ AP-HP, Department of Internal Medicine B, Lariboisière Hospital, Paris, France; ${ }^{6}$ Lebanese American University, School of Medicine, Beirut, Lebanon

*Correspondence: Dr C Julier, Inserm UMR-S958, Medical Faculty Paris 7, site Villemin, 10 avenue de Verdun, 75010 Paris, France. Tel: +33 157278543 ;

Fax: +33 1572785 54; E-mail: cecile.julier@inserm.fr

Received 14 November 2012; revised 11 February 2013; accepted 4 April 2013; published online 8 May 2013 
prediction was performed using NetGene2 (www.cbs.dtu.dk/services/ NetGene2), followed by RT-PCR and sequencing of amplified fragments. Primers and PCR conditions are available upon request.

\section{RESULTS}

\section{Patient's description}

Medical history and clinical observations. The patient was born to first-degree consanguineous parents. Decrease in visual acuity was noticed at the age of 3 years, and progressive vision loss with optic atrophy resulted in blindness at age 17 . Hearing impairment was noted at age 4, which required a hearing aid at age 17 . He had frequent seizures, difficulty walking, mild mental retardation with learning difficulties and recurring confusional episodes. Glycosuria was first noticed at age 3 , but was not further investigated or treated at that time. At age 13, the patient presented with severe ketoacidosis, HbAlc was $10.5 \%$, and he was treated with insulin subsequently. GAD autoantibodies were negative when tested at age 17. The combination of juvenile-onset insulin-dependent diabetes and optic atrophy led to the likely diagnosis of WFS, and at age 19 the patient participated in a comprehensive clinical study of WFS and underwent a series of clinical and biological tests. ${ }^{4}$

Clinical and imaging studies. Ophthalmic examination confirmed blindness, with no light perception. Fundoscopy showed posterior lens opacities, optic atrophy, sinusoidal arteries, central and peripheral retinal pigmentation suggesting retinitis pigmentosa. Optic atrophy was also confirmed by magnetic resonance imaging. He had abnormal extraocular movements with vertical nystagmus. Audiometry showed $70 \%$ of discrimination at $80 \mathrm{db}$ at the right and left ears. He had insulin resistance, with elevated fasting C-peptide at $10 \mathrm{ng} / \mathrm{ml} \mathrm{(N:0.8-}$ $4 \mathrm{ng} / \mathrm{ml}$ ), rising to $15.4 \mathrm{ng} / \mathrm{ml}$ after $1 \mathrm{mg}$ glucagon stimulation. Partial pituitary insufficiency was present, as evidenced by: (1) low morning cortisol level $(4.4 \mu \mathrm{g} / \mathrm{dl}, \quad N$ : 5.6-21.8) with non-elevated ACTH $(21 \mathrm{ng} / \mathrm{dl}, \mathrm{N}:$ 10-90), and absence of significant response after tetracosacide $(15 \mu \mathrm{g} / \mathrm{dl})$; (2) low IGF1 concentration $(72.5 \mathrm{ng} / \mathrm{ml}, N$ : $122-400)$ without hGH rise during insulin hypoglycemia $(2.5 \mathrm{ng} / \mathrm{ml})$; (3) low testosterone value $(2.15 \mathrm{ng} / \mathrm{ml}, N$ : 3-8) without increase of LH (4IU/l, N:1-8) and FSH (2.84 IU/l, N:4-10) indicating hypogonadotropic hypogonadism; (4) free T4 concentration was at the low normal range $(0.86 \mathrm{ng} / \mathrm{dl}, \mathrm{N}: 0.85-1.98)$ with normal TSH value $(2 \mathrm{mIU} / \mathrm{ml}, \mathrm{N}: 0.3-4)$; (5) central $\mathrm{ADH}$ deficiency with partial diabetes insipidus, as evaluated by hydric restriction test, showing maximal urine concentration of $470 \mathrm{mosm} / \mathrm{kg}(\mathrm{N}:>800 \mathrm{mosm} / \mathrm{kg})$, with positive response to exogenous $\mathrm{ADH}$ injection (Desmopressin) $(600 \mathrm{mosm} / \mathrm{kg})$. Cardiac echography and electrocardiogram were normal. Electroencephalogram showed occipital slowing, consistent with seizures. Neurological examination revealed peripheral neuropathy with loss of sensitivity to vibration, heat and cold. Recent memory was preserved and reasoning was normal except during confusional episodes. There was evidence of nephropathy, with creatinine clearance of $75 \mathrm{ml} / \mathrm{min}(\mathrm{N}: 100-140 \mathrm{ml} / \mathrm{min})$ and macroalbuminuria of $500 \mathrm{mg} / 24 \mathrm{~h}(\mathrm{~N}:<300 \mathrm{mg} / 24 \mathrm{~h})$. Hepatic evaluation showed normal SGPT and SGOT, but elevated GGT (203 IU/ml; $N$ : $<74 \mathrm{IU} / \mathrm{ml}$ ). Abdominal CT scan and echography disclosed an enlarged spleen $(18 \mathrm{~cm})$, and a dysmorphic liver with hypertrophy of the left lobe. Hepatic biopsy revealed a cirrhotic process and mild focal steatosis. Lipoprotein profile was normal except for slightly elevated triglycerides $(2.39 \mathrm{mmol} / \mathrm{l} ; N:<2.26 \mathrm{mmol} / \mathrm{l})$. Tests for viral hepatitis B and C were negative. BMI was $22.9(167 \mathrm{~cm} / 64 \mathrm{~kg})$ at age 19 , with no evidence of truncal obesity. The patient died at the age of 26; the cause of death was not determined.

\section{Genetic diagnosis}

Because of the association of juvenile-onset insulin-dependent diabetes, optic atrophy, deafness, diabetes insipidus and neurological manifestations, we first tested WFS1 as a candidate gene. Linkage analysis showed that the patient was heterozygous for the WFS1 region, and sequencing of WFS1 exons in the patient and his parents showed no mutation, therefore excluding WFS1 as the responsible gene (data not shown). We then considered other monogenic forms of syndromic diabetes, and selected genes that mapped to homozygous chromosome regions in the patient. This led us to select ALMS1, responsible for Alström syndrome, as a candidate gene; the ZCD2 gene, responsible for a rare variant form of WFS, ${ }^{8}$ was located in a heterozygous chromosome region of the patient and was thus excluded. By sequencing of ALMS1 exons and flanking regions, we identified a homozygous T/G substitution in intron 18, located 3 bases before the start of exon 19 (IVS18-3 T > G, Figure 1a). This variant was absent in 180 unrelated Lebanese population controls and in public variant databases. This variant is predicted to abolish the acceptor site located at the intron 18-exon 19 boundary that would result in exon 19 skipping. By RT-PCR on RNA from the parents and sequencing using primers located in exons 18 and 20, we confirmed that they were heterozygous for a shorter transcript lacking exon 19 (Figure 1b). The deletion of exon 19 causes a frameshift, resulting in a premature STOP codon and a truncated protein (V3958fs3964X, Figure 1c).

We then tested if ALMS1 mutations or variants may contribute to juvenile-onset diabetes (diagnosed as T1D) in Lebanon. Linkage analysis in 220 consanguineous, multiplex or extended Lebanese T1D families showed no evidence of linkage of T1D to the ALMS1 chromosome region (data not shown). We selected 11 families compatible with linkage to this region, and sequenced all the coding exons of the ALMS1 gene and their flanking regions, and did not find any mutation. We conclude that ALMS1 mutations are not a frequent cause of diabetes diagnosed as T1D in Lebanon.

\section{DISCUSSION}

In this study, we identified a novel ALMS1 splice mutation in a syndromic diabetes patient with clinical manifestations of Wolfram and Alström syndromes. ${ }^{9-11}$ Clinical characteristics of these syndromes and of the patient are shown in Table 1. Both syndromes show extensive clinical variability and share impaired glucose metabolism (diabetes), impaired vision and hearing, and neurological abnormalities, which were observed in our patient. Compared with Alström syndrome, he had several atypical features, which are more characteristic of WFS: the presence of juvenile-onset insulin-dependent diabetes with ketoacidosis at onset, optic atrophy, normal weight, stature and BMI, and near-normal lipid profile. In addition, he suffered from peripheral neuropathy, which has been reported to be remarkably absent in Alström patients. ${ }^{12}$ However, the early-onset retinitis pigmentosa and complete blindness by age 17, the early-onset hearing impairment, insulin resistance, cirrhosis and liver steatosis, and splenomegaly were suggestive of Alström syndrome. In the absence of many key features of Alström syndrome, this diagnosis was not evoked prior to the genetic study. Absence of obesity ${ }^{13,14}$ and normal adult stature are exceptional in Alström syndrome (Table 1); similarly, insulin-dependent diabetes with ketoacidosis at onset ${ }^{15}$ and diabetes insipidus ${ }^{16}$ have each been reported in a single patient. The association of these very unusual features makes this case particularly remarkable.

To date, more than 100 mutations have been identified in the ALMS1 gene, all causing Alström syndrome, most of them nonsense 


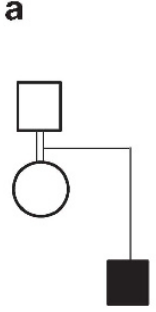

Control
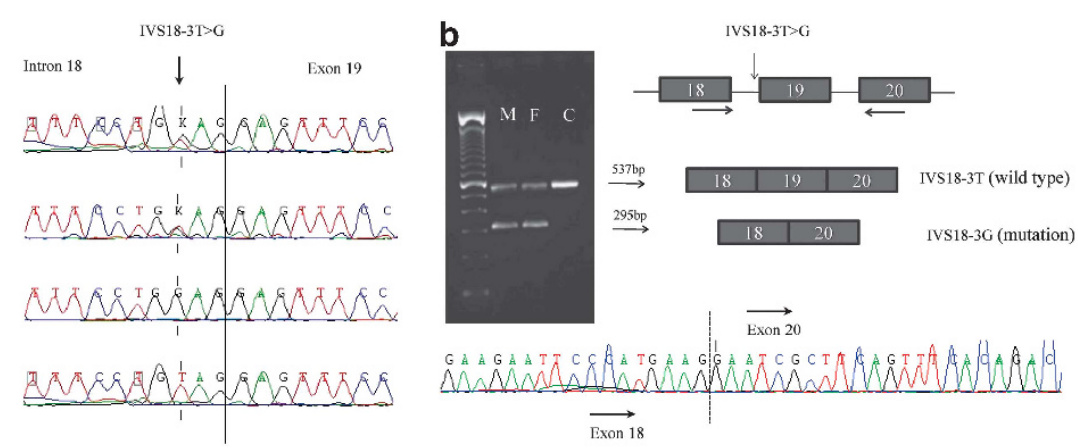

C

ALMS1 gene

IVS18-3T>G

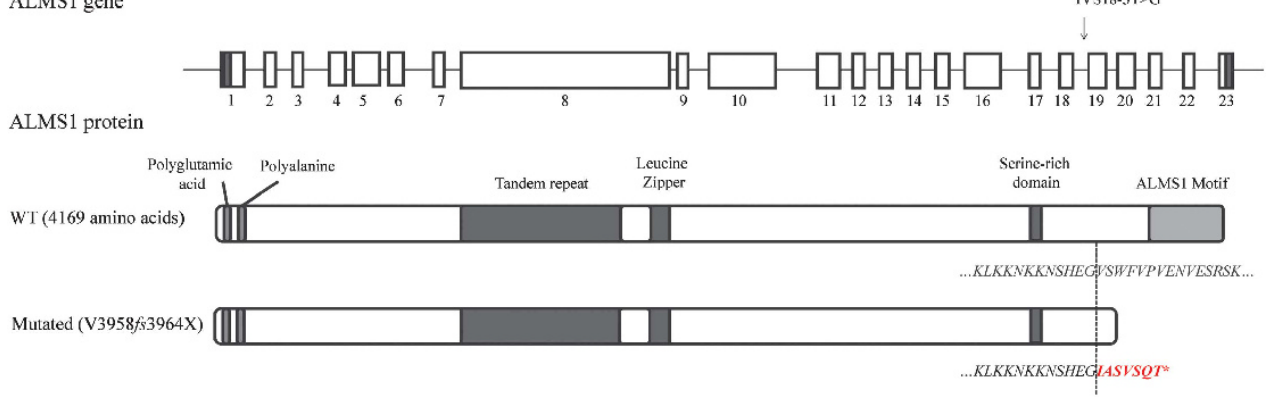

Figure 1 Identification of a novel homozygous splice mutation in the ALMS1 gene and its consequences. (a) Sequencing results of the parents, affected child and control, showing a predicted splice mutation (arrow) $3 \mathrm{bp}$ before the start of exon 19. (b) RT-PCR using forward and reverse primers (arrows) performed on mRNA from parents and a control (M: mother, F: father, C: control), demonstrating skipping of exon 19 in the mutated allele. (c) Schematic representation of the ALMS1 gene, protein and domains, in wild type (WT) and mutated form. Protein structure and domains are based on Girard and Petrovsky. ${ }^{25}$

Table 1 Clinical comparison between Alström syndrome, Wolfram syndrome and patient's characteristics

\begin{tabular}{|c|c|c|c|c|}
\hline & Clinical Feature & Alström syndrome & Wolfram Syndrome & Patient \\
\hline Eye & Cone rod dystrophy/retinal pigmentosis & $+(100 \%)$ & - & Retinal pigmentosis \\
\hline & Optic atrophy & - & $+(100 \%)$ & Optic atrophy \\
\hline Hearing & Sensorineural hearing loss & + & + & Sensorineural hearing loss \\
\hline \multirow[t]{2}{*}{ Obesity/ stature } & Truncal obesity & $+(98 \%)$ & - & Absent $(\mathrm{BMI}=22.9)$ \\
\hline & Short stature (adult, $<5$ th percentile) & $+(98 \%)$ & - & Absent (height: $167 \mathrm{~cm}$ ) \\
\hline Diabetes & Diabetes insipidus & $-{ }^{a}$ & + & Partial diabetes insipidus \\
\hline Dermatology & Acanthosis nigricans & + & - & Absent \\
\hline Hypogonadism & Male hypogonadism & + & + & Hypogonadotropic hypogonadism \\
\hline Lipids & Hypertriglyceridemia/hypercholesterolemia & + & - & Slightly elevated triglycerides \\
\hline \multirow[t]{2}{*}{ Hepatic } & Cirrhosis and steatosis & + & - & Cirrhosis and focal steatosis \\
\hline & Hepatosplenomegaly & + & - & Splenomegaly \\
\hline Cognition & Developmental delay & + & - & Mild mental retardation \\
\hline \multirow[t]{2}{*}{ Neurological } & Seizures & + & + & Seizures \\
\hline & Peripheral Neuropathy & - & + & Peripheral neuropathy \\
\hline Skeletal & Skeletal deformities & + & - & Kyphosis \\
\hline
\end{tabular}

Clinical descriptions for Alström and Wolfram syndromes are compiled from Barret et al. ${ }^{9}$ and Marshall et al. ${ }^{10,11}+$ : frequent occurrence of the trait; -: absence of the trait. Frequencies of the

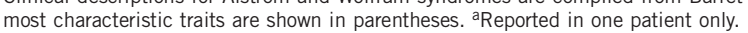

or frameshift mutations located in exons $5-18 .^{10,17}$ The mutation identified in our patient, IVS18-3T $>$ G, is very similar to a splice mutation (IVS18-2A $>$ T) that has been reported in two Saudi Arabian sibs diagnosed with Alström syndrome, presenting obesity and short stature. $^{18}$ So, except for the possible exception of dilated cardiomyopathy (DCM), which was absent from these three 
patients, other atypical features in our patients are unlikely to depend on the nature of the mutation. Interestingly, DCM was one of the Alström traits showing suggestive evidence for genotype-phenotype correlation with increased risk associated with mutations located in exon $16 .^{19}$

Most studies searching for ALMS1 mutations have targeted patients who had been diagnosed with Alström syndrome based on their clinical presentation, which is unlikely to detect very atypical cases. In contrast, we studied a patient who was not clinically diagnosed with Alström syndrome. Consequently, our study extends the phenotypic variability of ALMS1 mutations and suggests that it may be broader than currently thought. Similarly, we previously identified novel hypomorphic mutations in monogenic diabetes genes by studying patients who did not show the typical features of the corresponding syndrome, as in the case of the WFS1 gene in T1D in Lebanon ${ }^{3}$ and of the PDX1 gene in neonatal diabetes. ${ }^{20}$

Our study raises the issue of the accuracy of clinical diagnosis of rare diabetes syndromes, especially when they are very variable. In the present case, the clinical diagnosis was further challenged by the conjunction of three factors: the relatively high prevalence of WFS in Lebanon, therefore well known to clinicians; the rarity of Alström syndrome in Lebanon, this patient being the first reported case in Lebanon; and the atypical Alström presentation in this patient.

Our observation led us to question if ALMS1 mutations may explain a subset of patients diagnosed as T1D. Our linkage and sequencing studies in T1D families in Lebanon do not support this hypothesis, and large-scale linkage and association studies in outbred Caucasian T1D families ${ }^{21-23}$ did not show any signal at ALMS1, excluding a significant contribution of common or rare variants of the ALMS1 gene to T1D risk.

Several hypotheses may be proposed to explain the atypical clinical features in our patient. In view of the limited number of splice or missense variants reported in Alström patients to date, ${ }^{10}$ we cannot exclude that a subset of these may result in atypical Alström presentation. Additional modifier effects or risk factors may also modulate disease presentation, as suggested by the variable Alms1 knockout mouse phenotype depending on their genetic background. ${ }^{10}$ Interestingly, we determined that this patient was HLA-DRB1 ${ }^{*} 04$ positive, and therefore carrier of an independent T1D susceptibility, which may have potentiated insulin dependency in an ALMS1-mutated genetic background.

Overall, our observation suggests that Alström syndrome, like other rare monogenic forms of diabetes, may be underdiagnosed, as a consequence of its rarity and the variability of the clinical presentation. For this and other rare monogenic diabetes syndromes, the use of systematic genetic diagnosis may reveal the true prevalence and extent of variability of this and clinically overlapping syndromes. ${ }^{24}$ This would be particularly important to consider in populations where the prevalence of consanguinity or endogamy is high.

\section{CONFLICT OF INTEREST}

The authors declare no conflict of interest.

\section{ACKNOWLEDGEMENTS}

We thank the patient and his family for agreeing to participate in this study. We thank the Chronic Care Centre, Beirut, Lebanon, for their collaboration and support. This study was supported by institutional funds from Inserm and University Paris 7, a joint grant from the Juvenile Diabetes Research Foundation, the Fondation pour la Recherche Médicale and Inserm and a grant from the Agence Nationale de la Recherche to CJ. We thank the AP-HP for their support.

1 American_Diabetes_Association. Diagnosis and classification of diabetes mellitus. Diabetes Care 2011; 34:Suppl 1 S62-S69.

2 Barrett TG: Differential diagnosis of type 1 diabetes: which genetic syndromes need to be considered? Pediatr Diabetes 2007; 8: 15-23.

3 Zalloua PA, Azar ST, Delepine M et al: WFS1 mutations are frequent monogenic causes of juvenile-onset diabetes mellitus in Lebanon. Hum Mol Genet 2008; 17: 4012-4021.

4 Medlej R, Wasson J, Baz P et al: Diabetes mellitus and optic atrophy: a study of Wolfram syndrome in the Lebanese population. J Clin Endocrinol Metab 2004; 89: 1656-1661.

5 Zalloua PA, Xue Y, Khalife J et al: Y-chromosomal diversity in Lebanon is structured by recent historical events. Am J Hum Genet 2008; 82: 873-882.

6 Takahashi M, Matsuda F, Margetic N, Lathrop M: Automated identification of single nucleotide polymorphisms from sequencing data. J Bioinform Comput Biol 2003; 1 : 253-265.

7 Abecasis GR, Cherny SS, Cookson WO, Cardon LR: Merlin-rapid analysis of dense genetic maps using sparse gene flow trees. Nat Genet 2002; 30: 97-101.

$8 \mathrm{Amr} \mathrm{S}$, Heisey C, Zhang $\mathrm{M}$ et al: A homozygous mutation in a novel zinc-finger protein, ERIS, is responsible for Wolfram syndrome 2. Am J Hum Genet 2007; 81: 673-683.

9 Barrett TG, Bundey SE, Macleod AF: Neurodegeneration and diabetes: UK nationwide study of Wolfram (DIDMOAD) syndrome. Lancet 1995; 346: 1458-1463.

10 Marshall J, Maffei P, Collin G, Naggert J: Alstrom syndrome: genetics and clinical overview. Curr Genomics 2011; 12: 225-235.

11 Marshall J, Paisey R, Carey C, Macdermott S: Alstrom Syndrome; in: Pagon RA BT, Dolan CR, Stephens K, Adam MP (ed) GeneReviews [Internet]. Seattle (WA): University of Washington, Seattle, 2003 [Updated 2012 May 31]).

12 Paisey RB, Paisey RM, Thomson MP et al: Protection from clinical peripheral sensory neuropathy in Alström syndrome in contrast to early-onset type 2 diabetes. Diabetes Care 2009; 32: 462-464.

13 Koc E, Bayrak G, Suher M, Ensari C, Aktas D, Ensari A: Rare case of Alstrom syndrome without obesity and with short stature, diagnosed in adulthood. Nephrology (Carlton) 2006; 11: 81-84

14 Tasdemir S, Guzel-Ozanturk A, Marshall J et al: Atypical presentation and a novel mutation in ALMS1: implications for clinical and molecular diagnostic strategies for Alstrom syndrome. Clin Genet 2013; 83:1 96-98.

15 Satman I, Yilmaz MT, Gürsoy $\mathrm{N}$ et al: Evaluation of insulin resistant diabetes mellitus in Alström syndrome: a long-term prospective follow-up of three siblings. Diabetes Res Clin Pract 2002; 56: 189-196.

16 Aynaci F, Okten A, Mocan H, Gedik Y, Sarpkaya A: A case of Alström syndrome associated with diabetes insipidus. Clin Genet 1995; 48: 164-166.

17 Joy T, Cao H, Black G et al: Alstrom syndrome (OMIM 203800): a case report and literature review. Orphanet Journal of Rare Diseases 2007; 2: 49.

18 Aldahmesh MA, Abu-Safieh L, Khan AO et al: Allelic heterogeneity in inbred populations: The Saudi experience with Alström syndrome as an illustrative example. Am J Med Genet Part A 2009; 149A: 662-665.

19 Marshall J, Hinman E, Collin G et al: Spectrum of ALMS1 variants and evaluati on of genotype-phenotype correlations in Alstrom syndrome. Hum Mutat 2007; 28: 1114-1123.

20 Nicolino M, Clairborn K, Senée V, Boland A, Stoffers D, Julier C: A novel hypomorphic PDX1 mutation responsible for Permanent Neonatal Diabetes with subclinical exocrine deficiency. Diabetes 2009; 59: 733-740.

21 Concannon P, Chen W-M, Julier C et al: Genome-wide scan for linkage to type 1 diabetes in 2496 multiplex families from the Type 1 Diabetes Genetics Consortium. Diabetes 2009; 58: 1018-1022.

22 Morahan G, Mehta M, James I et al: Tests for genetic interactions in type 1 diabetes: linkage and stratification analyses of 4422 affected sib-pairs. Diabetes 2011; 60:3 1030-1040.

23 Barrett JC, Clayton DG, Concannon P et al: Genome-wide association study and metaanalysis find that over 40 loci affect risk of type 1 diabetes. Nat Genet 2009; 41 : 703-707.

24 Redin C, Le Gras S, Mhamdi 0 et al: Targeted high-throughput sequencing for diagnosis of genetically heterogeneous diseases: efficient mutation detection in Bardet-Biedl and Alström syndromes. J Med Genet 2012; 49: 502-512.

25 Girard D, Petrovsky N: Alstrom syndrome: insights into the pathogenesis of metabolic disorders. Nat Rev Endocrinol 2011; 7: 77-88. 